\title{
Subjective perceptions of load carriage on the head and back in Xhosa women
}

\author{
R. Lloyd ${ }^{1 *}$, B. Parr ${ }^{2}$, S. Davies ${ }^{2}$ and C. Cooke ${ }^{3}$ \\ ${ }^{1}$ Division of Sport and Exercise Sciences, University of Abertay Dundee, Dundee, DD1 1HG, UK \\ ${ }^{2}$ Department of Sports Management, Cape Peninsula University of Technology, PO Box 652, Cape \\ Town, 8000, South Africa \\ ${ }^{3}$ Carnegie Research Institute, Leeds Metropolitan University, Leeds, LS6 3QS, UK \\ * Author for correspondence - r.lloyd@ abertay.ac.uk
}

\begin{abstract}
The purpose of this study was to compare the subjective perceptual responses to both head-loading and back-loading in a group of Xhosa women. Thirty two women were divided into three groups based on their experience of head-loading and walked on a treadmill on two occasions, head-loading and back-loading, at a self selected walking speed for four minutes with a variety of loads until pain or discomfort caused the test to be terminated or a load of $70 \%$ body mass was successfully carried. After each workload there was a one minute rest period during which the women indicated feelings of pain or discomfort in particular areas of the body via visual analogue scales. At the end of each test the women were asked to complete further questionnaires relating to pain and discomfort and on completion of the second test were also asked to compare the two loading conditions. Finally the women were interviewed to establish their history of load carriage and associated pain and discomfort. The data indicate that whilst back-loading was generally associated with more areas of discomfort than head-loading, the pain and discomfort in the neck associated with head-loading was the predominant factor in the termination of tests and that this was independent of head-loading experience. This early termination meant that, on average, the women could carry greater loads on their backs than on their heads. The study suggests that further work needs to be carried out to establish viable alternatives to head-loading for rural dwellers in Africa.
\end{abstract}

Keywords: Load carriage, subjective perceptions, head-loading, African women 


\section{Introduction}

The study of human load carriage remains an important area of investigation, with much of the recent work relating to either the physiology (e.g. Abe et al, 2008; Bastien et al, 2005) or the biomechanics (e.g. Sharpe et al, 2008; LaFiandra et al, 2003) of load carriage. It has been argued, however, that subjective perceptions are an important area of study when considering means and methods of load carriage (Legg et al, 1997), partly because they are important in choosing a load carrying method (Mackie and Legg, 2008) and partly because they may assist in differentiating between load carriage methods when physiological and biomechanical differences are not large enough to distinguish between them (Legg et al, 1997). A number of methods have been employed to assess subjective perceptual responses to load carriage including whole body Rating of Perceived Exertion (RPE), either 20 point (e.g. Holewijn et al, 1992) or 10 point (e.g. Stuempfle et al, 2004), Differentiated RPE (e.g. Kirk and Schneider, 1992), Category Rating Scales (CRS) (e.g. Mackie et al, 2003), Visual Analogue Scales (VAS) (e.g. Ling et al, 2004), post test questionnaires (e.g. Whitfield et al, 2005) and interviews (e.g. Birrell and Hooper, 2007). Legg et al (1997) have argued that the use of post test questionnaires and VAS is superior to in-test CRS in the ability to adequately differentiate between load carriage means and methods. In light of this the present study will employ in-test VAS, post test questionnaires and semi structured interviews as methods of assessing subjective perceptions.

The carrying of relatively heavy loads remains a necessity for a number of different groups, particularly schoolchildren, hikers and the military and this is reflected in the load carriage literature with a number of recent studies employing subjective perceptual assessments concentrating on schoolchildren (Mackie et al, 2003; Whitfield et al, 2005; Mackie and Legg, 2008), hikers (Legg et al, 1997; Legg et al, 2003) and the military (Ling et al, 2004; Birrell and Hooper, 2007). There remains, however, one extremely large group for whom the 
carrying of heavy loads is a daily necessity and who are significantly under represented in the literature, rural dwellers in the developing world. For a variety of reasons, historical, political, social and economic, these rural dwellers typically use traditional means for carrying heavy loads such as water and wood. In Africa particularly this work falls mainly to the women and the dominant form of load carriage is head-loading, either directly or via a forehead strap. The issue of head load carriage has received little attention in the literature, mainly concentrating on the metabolic cost of the activity. Such data as does exist is somewhat contradictory and confounded by very small sample sizes. On the one hand Maloiy et al (1986), Charteris et al (1989a,b), and Nag and Sen (1978) have all presented data, based on samples of 4-6 participants, suggesting that head-loading is an extremely efficient method of load carriage whilst on the other hand Datta and Ramanathan (1971) and Das and Saha (1966) have argued, based on samples of 6 and 7 participants, that it is less efficient than back-loading. More recently, when considering load carriage by Nepalese porters, who use the head strap method, Malville et al. (2001) and Minetti et al. (2006) reached contrasting conclusions in relation to the economy of the method. In light of this contradictory data, the argument of Legg et al (1997) in relation to the discriminatory power of perceptual assessments and the fact that to date no subjective perceptual data is available in relation to head-loading, the purpose of this study was to investigate subjective perceptual responses to both head and back-loading in a group of Xhosa women. 


\section{Methods}

\subsection{Participants}

The sample for the study was drawn from the Xhosa people who are indigenous to the Eastern Cape region of South Africa. $17.6 \%$ of the South African population speak isiXhosa as a first language (South African Census 2001). Thirty two Xhosa women, thirteen with at least ten years experience of head load carriage (EXP), eleven with no experience of head load carriage (NON) and eight with some experience in childhood but no recent experience (INT), were recruited to take part in the study. Participants were recruited from amongst the student body and from the township of Khayelitsha on the outskirts of Cape Town. All participants gave informed consent for their participation in the study which had received ethical approval through standard institutional review procedures at both the University of Abertay Dundee and Cape Peninsula University of Technology. A summary of participant characteristics is presented in Table 1. One way ANOVA indicated no significant differences between the two groups for any of the parameters.

Table 1. Participant characteristics (Mean $\pm S D$ )

\begin{tabular}{|c|c|c|c|c|}
\hline & Age (years) & Stature $(\mathrm{m})$ & Mass $(\mathrm{kg})$ & Walking Speed $\left(\mathrm{km}^{\prime} \mathrm{h}^{-1}\right)$ \\
\hline \hline EXP & $22.5 \pm 2.1$ & $1.59 \pm 0.05$ & $66.0 \pm 12.9$ & $3.15 \pm 0.45$ \\
\hline INT & $23.1 \pm 3.9$ & $1.61 \pm 0.04$ & $66.5 \pm 12.6$ & $2.78 \pm 0.50$ \\
\hline NON & $21.2 \pm 2.4$ & $1.58 \pm 0.05$ & $66.8 \pm 14.5$ & $3.01 \pm 0.30$ \\
\hline P & 0.287 & 0.488 & 0.990 & 0.191 \\
\hline
\end{tabular}

\subsection{Experimental procedures}

The women each attended the Human Performance laboratory at Cape Peninsula University of Technology on three separate occasions. On the first occasion participants were screened for any potential contraindications to exercise, stature and mass were assessed, and questionnaires were completed relating to load carriage history. The women were then shown the twelve body pictures (e.g. Figure 1) that were to be used to record perceptions of pain and discomfort. The associated Visual Analogue Scales (VAS) and the whole body RPE scale (Borg 1982) were explained to them. The women were then habituated to the experimental protocol. A typical habituation session lasted between twenty and thirty minutes and involved the women, who for the most part had not been on a 
treadmill before, walking on the treadmill at various speeds until they felt comfortable with the experience. In addition they also tried out the two load carrying devices, a standard 451 backpack with hip belt (Karrimor, SA) for back loading and a plastic crate for head loading (the crate placed either directly on the head or on a small piece of rolled cloth to provide some cushioning), with and without loads. At the end of the session the women were asked to walk on the treadmill at a speed that they felt would be comfortable when carrying a heavy load. The chosen speeds (Table 1) were not significantly different $(\mathrm{P}=0.191$, one way ANOVA) and were similar to speeds employed in other comparable studies (Maloiy et al. 1986, Charteris et al. 1989a). The chosen walking speed of each participant was noted and used for the subsequent experimental trials.

On arrival at the laboratory at the next visit each participant chose at random, via the picking of a suitably marked piece of paper from a hat, the loading method for the first experimental trial. This involved walking, at the previously determined speed, for four minutes unloaded and then, after a one minute rest, a load of $10 \%$ body mass was added which was carried for a further four minutes. After a further rest of one minute the load was increased to $15 \%$ and carried for four minutes. This pattern was repeated with loads of $20 \%, 25 \%, 30 \%, 40 \%, 50 \%, 60 \%$ and $70 \%$ of body mass or until pain and discomfort led to voluntary cessation of the session. The load was calculated based on the body mass at the habituation session and was made up of the mass of the actual carrying device plus appropriate weightlifting plates, (between $2.5 \mathrm{~kg}$ and $10 \mathrm{~kg}$ ), and $100 \mathrm{~g}$ sandbags, which allowed the load to be adjusted to within $50 \mathrm{~g}$ of the required load. Each participant attended the laboratory one week later to repeat the experiment with the other loading device.

On occasions when the backpack was employed it was fitted to the participant and adjusted to ensure appropriate seating of the hip belt and shoulder straps. The crate used for head load carriage was suspended from a rig and lowered onto the participant so as to ensure that there was sufficient slack in the system to provide no support for the load but to allow the participant to drop from under the load safely in an emergency.

\subsection{Data collection and analysis}


During the one minute rest periods between each load participants were shown the 20 point RPE scale and asked to rate how they were feeling before being shown a series of six sheets containing two body pictures, where a different area of the body had been clearly shaded on each picture (e.g. Figure 1) and a 100mm VAS added below each image with anchor points of 'No Pain' at one end and 'Pain as bad as it could possibly be' at the other end (Hinnant 1994). Body pictures were shown in the same order on each occasion, starting with the neck and shoulders and working down the body to the feet. The sheets were laminated and the participants asked to mark each VAS using a fine point, washable, marker pen to indicate the pain/discomfort they were feeling. The marked point on each scale was then measured to the nearest millimetre to provide a pain score for each identified body region at each load. These were subsequently analysed using a MANOVA with repeated measures (SPSS, v17.0). Analysis considered scores for loads of 0-20\% BM, the range completed by all participants. A secondary analysis of scores at the penultimate and final load for each participant was also undertaken using MANOVA with repeated measures since this reflected the same relative loads and also gave an insight into both the determinants of test cessation and also the pattern of scores. In all cases significant main effects were followed up via pairwise comparisons with Bonferroni adjustment whilst significant interactions were explored via 95\% Confidence Intervals of cell means. RPE scores were compared via repeated measures ANOVA and significant differences followed up using the same procedures as described above.

Previously Mackie et al (2003) have suggested that the actual scores given by individuals may reflect certain psychological traits such as willingness to use extreme scores and consequently analysed similar data using both raw scores and $\mathrm{z}$ scores, finding no material difference in outcome. Given the repeated measures approach taken here, where the point of comparison is effectively intra participant, and accepting that absolute values will reflect such psychological traits as well as individual sensitivity to, and tolerance of, pain, it was felt appropriate to work with raw scores. At the end of each experimental trial a questionnaire was administered which asked participants to rate the comfort, freedom of movement and stability of the loading method associated with that trial using a seven point Likert scale and provided space for individual comments. The quantitative data was subsequently 
analysed using an ANOVA with repeated measures. The questionnaire also asked participants to indicate, by choosing from a list, any areas of the body where pain/discomfort had been felt during that trial. At the end of the final trial an additional questionnaire was administered which asked participants to choose between head- and back- loading in terms of comfort, stability and freedom of movement as well as indicating which loading method was associated with most discomfort in each of the body areas covered by the in-test VAS. Finally all participants with experience of head-loading participated in a semi-structured interview designed to explore their perceptions of head-loading and understand how they had learnt the loading method. Interviews typically lasted fifteen minutes.

Maximum load carried was recorded and compared between the groups and conditions by means of a further ANOVA with repeated measures. One way ANOVA was used to assess differences in physical characteristics and walking speed between the groups.
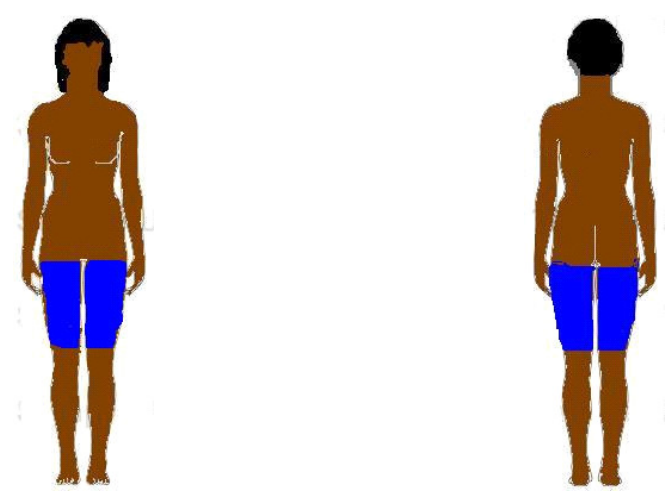

On the Scale below indicate the amount of pain/discomfort you feel in the area indicated

Front of Thigh

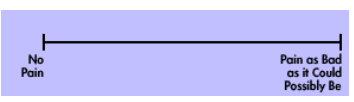

Back of Thigh

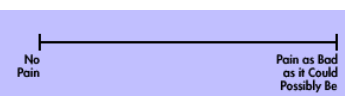

Figure 1. Sample VAS data collection sheet (Quadriceps and Hamstrings) 


\section{Results}

\subsection{Maximum Load Carried}

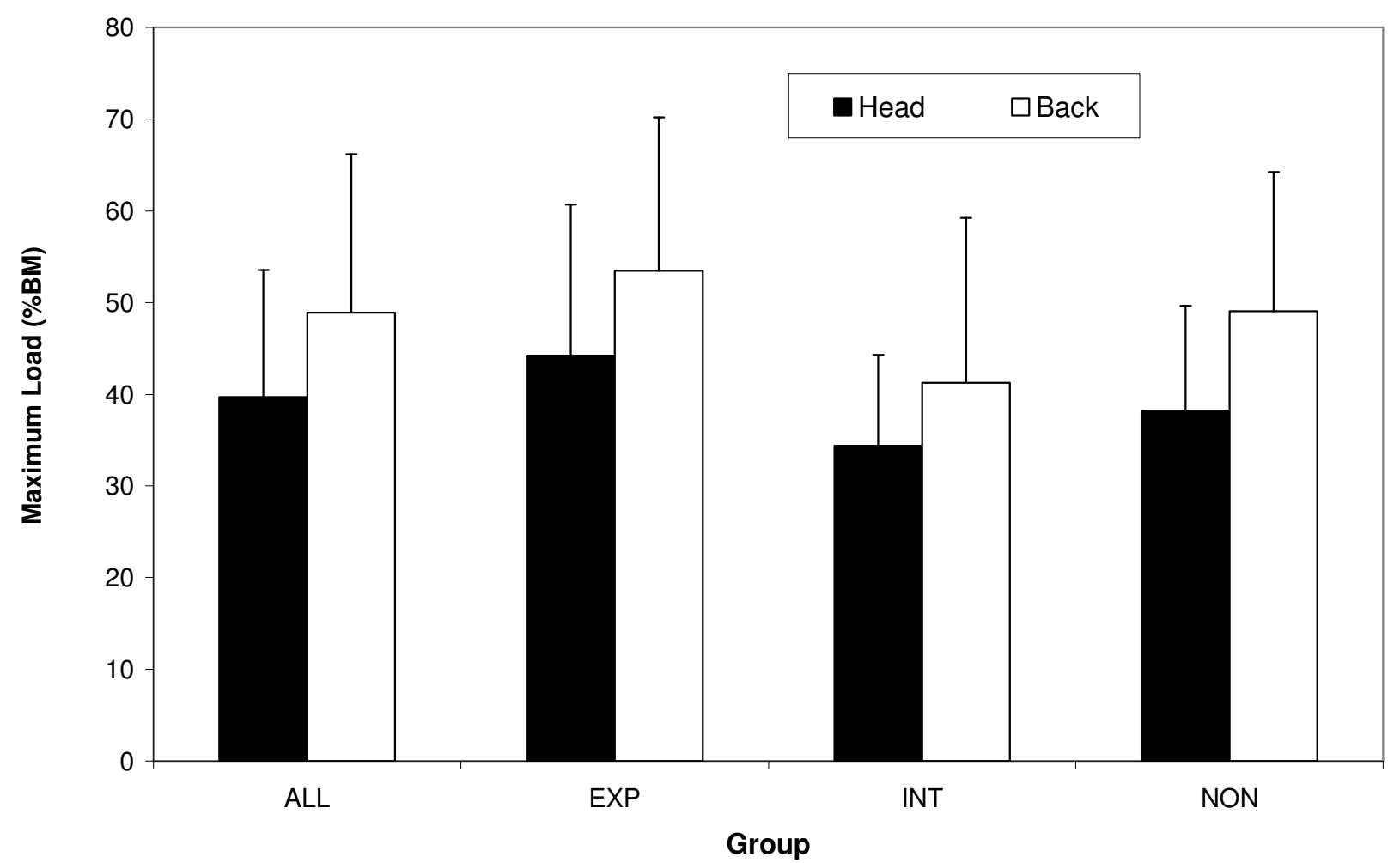

Figure 2. Mean $(+s)$ maximum load carried in each of the loading conditions for each group

On average the load that could be carried on the back was $9.2 \% \mathrm{BM}(6.1 \mathrm{~kg})$ greater than on the head $(26.4 \pm 9.2 \mathrm{~kg}$ v $32.5 \pm 11.5 \mathrm{~kg}$ for head and back respectively, $\mathrm{P}=0.007)$. There was no difference in the loads carried by each group $(\mathrm{P}=0.181)$ nor, as is clear from Figure 2, was there any interaction between group and loading method $(\mathrm{P}=0.879)$. Overall, only seven participants carried the same load in both conditions (five EXP and two INT), nine carried more on the head (two EXP, two INT and five NON) whilst sixteen (six EXP, four INT and six NON) carried more on the back. Of the thirty two participants only two carried $70 \%$ in both conditions, one carried $60 \%$, six carried $50 \%$, five carried $40 \%$, ten carried $30 \%$, five carried $25 \%$ and three carried $20 \%$. Considering the individual conditions, for head-loading only two achieved a maximum load of $70 \% \mathrm{BM}$, two achieved $60 \%$, eight achieved 50\%, six achieved 40\%, eight achieved 30\%, five achieved $25 \%$ and one achieved $20 \%$. For back-loading the figures were nine with $70 \%$, four with $60 \%$, five with $50 \%$, five with $40 \%$, six with $30 \%$, one with $25 \%$ and two with $20 \%$. Given the drop off in participant numbers beyond a 
load of $20 \%$ BM the subsequent analyses either refer to end point loads in each condition or to loads of up to $20 \%$ BM.

\subsection{Pain scores at $0-20 \%$ BM}

Considering, for all participants, the main effect of loading condition, overall RPE scores were similar for both loading methods $(9.6 \pm 2.1$ v 9.4.0 \pm 3.1 for head and back load respectively, $\mathrm{P}=0.667$ ). Considering the VAS data via MANOVA there was no significant difference between the two loading conditions (total pain scores $33.4 \pm 100.0$ v $43.4 \pm 109.4$ for head and back load respectively, $\mathrm{P}=0.654$ ) (see table 1 for indicative data at $20 \% \mathrm{BM}$ only). There was, however, a significant main effect for body area $(\mathrm{P}=0.001)$ with scores associated with the neck, shoulders, upper back and calf all being significantly greater than all other body areas. There was also a significant interaction between loading condition and body area $(\mathrm{P}=0.01)$, with the score associated with the neck being significantly greater in the head-loading condition than in the back loading condition (95\% CIs $7.0-13.8 v-0.3-5.6$ for head and back loading respectively) and a trend for difference between the two conditions for calf pain (95\% CIs $-0.8-4.1$ v $3.8-11.5$ for head and back loading respectively). There was no overall difference between the groups $(\mathrm{P}=0.193)$ although there was a significant interaction between body area and group $(\mathrm{P}=0.018)$ resulting from a significantly greater neck pain score for the INT group (95\% CI 7.8-17.2) than either the EXP $(0.9-7.8)$ or the NON group (-0.8 -

6.7) (figure 3). There was also a tendency for the INT to report higher pain scores for the shoulder and calf areas. The pattern of pain scores was largely consistent across the condition $\mathrm{x}$ group interaction $(\mathrm{P}=0.561)$ The pattern of response across the load $\mathrm{x}$ group interaction was also consistent $(\mathrm{P}=0.249)$. Overall pain scores increased with increasing load $(\mathrm{P}=0.005)$ although this was not consistent across all body areas, (load $\mathrm{x}$ body area interaction $\mathrm{P}<0.0005)$. Pain scores associated with the chest, hips, buttocks and feet only exhibited significant change between the $15 \%$ and $20 \%$ loads whilst pain scores for all other areas rose significantly with increasing load. The pattern of response to increasing load was consistent across the two loading conditions $(\mathrm{P}=0.588)$. 
Table 2 below shows data for VAS scores at $20 \%$ BM, the final load completed by all participants in both conditions. Analysis of the data is consistent with that reported above with no significant difference between loading conditions $(\mathrm{P}=0.976)$, a significant difference between body areas (neck, shoulders and upper back significantly greater than all other body areas except calves, $\mathrm{P}=0.003)$ and a significant interaction between body area and loading condition $(\mathrm{P}=0.009)$. There was no significant difference between the groups $(\mathrm{P}=0.363)$, although there was a significant interaction between body area and group $(\mathrm{P}=0.038)$.

Table 2. Mean $(+s)$ pain scores at final load for each group at a load of $20 \%$ BM in both conditions. (a indicates significant difference between conditions $(P<0.05)$, $b$ indicates trend for difference between conditions $(P<0.10)$ and $c$ indicates significant difference between INT group and both EXP and $\operatorname{NON}(P<0.05))$

\begin{tabular}{|c|c|c|c|c|c|c|c|c|}
\hline & \multicolumn{2}{|c|}{ Whole Group } & \multicolumn{2}{|c|}{ EXP } & \multicolumn{2}{|c|}{ INT } & \multicolumn{2}{|c|}{ NON } \\
\hline & Head & Back & Head & Back & Head & Back & Head & Back \\
\hline RPE & $\begin{array}{l}12.5 \\
(2.5)\end{array}$ & $\begin{array}{l}12.0 \\
(3.4)\end{array}$ & $\begin{array}{l}12.5 \\
(2.6)\end{array}$ & $\begin{array}{l}11.9 \\
(2.9)\end{array}$ & $\begin{array}{l}12.4 \\
(3.3)\end{array}$ & $\begin{array}{l}13.0 \\
(4.2)\end{array}$ & $\begin{array}{l}12.5 \\
(1.9)\end{array}$ & $\begin{array}{l}11.7 \\
(4.0)\end{array}$ \\
\hline Neck & $\begin{array}{l}20.3^{a} \\
(25.4)\end{array}$ & $\begin{array}{c}7.7 \\
(21.6)\end{array}$ & $\begin{array}{c}16.3 \\
(28.8)\end{array}$ & $\begin{array}{c}10.1 \\
(21.1)\end{array}$ & $\begin{array}{l}40.0^{c} \\
(24.5)\end{array}$ & $\begin{array}{c}18.0 \\
(40.2)\end{array}$ & $\begin{array}{c}16.0 \\
(19.6)\end{array}$ & $\begin{array}{c}0.3 \\
(1.0)\end{array}$ \\
\hline Shoulders & $\begin{array}{c}16.0 \\
(22.8)\end{array}$ & $\begin{array}{c}17.3 \\
(24.5)\end{array}$ & $\begin{array}{c}14.5 \\
(27.5)\end{array}$ & $\begin{array}{c}16.8 \\
(22.4)\end{array}$ & $\begin{array}{c}11.6 \\
(16.4)\end{array}$ & $\begin{array}{c}33.0 \\
(43.3)\end{array}$ & $\begin{array}{c}19.9 \\
(21.4\end{array}$ & $\begin{array}{c}10.7 \\
(10.5)\end{array}$ \\
\hline Upper Back & $\begin{array}{c}15.6 \\
(20.0)\end{array}$ & $\begin{array}{c}10.8 \\
(15.3)\end{array}$ & $\begin{array}{c}21.0 \\
(25.2)\end{array}$ & $\begin{array}{c}12.1 \\
(15.6)\end{array}$ & $\begin{array}{c}20.0 \\
(18.6)\end{array}$ & $\begin{array}{c}17.8 \\
(22.2)\end{array}$ & $\begin{array}{c}7.3 \\
(8.7)\end{array}$ & $\begin{array}{c}6.2 \\
(6.0)\end{array}$ \\
\hline Chest & $\begin{array}{c}3.0 \\
(12.1)\end{array}$ & $\begin{array}{c}4.0 \\
(9.8)\end{array}$ & $\begin{array}{c}4.7 \\
(16.9)\end{array}$ & $\begin{array}{c}2.6 \\
(7.4)\end{array}$ & $\begin{array}{c}0.0 \\
(0.0)\end{array}$ & $\begin{array}{c}7.0 \\
(15.7)\end{array}$ & $\begin{array}{c}2.3 \\
(0.0)\end{array}$ & $\begin{array}{c}4.3 \\
(10.7)\end{array}$ \\
\hline Abdomen & $\begin{array}{c}3.3 \\
(16.9)\end{array}$ & $\begin{array}{c}4.2 \\
(11.7)\end{array}$ & $\begin{array}{c}7.0 \\
(25.20\end{array}$ & $\begin{array}{c}5.9 \\
(12.6)\end{array}$ & $\begin{array}{c}0.0 \\
(0.0)\end{array}$ & $\begin{array}{c}9.0 \\
(20.1)\end{array}$ & $\begin{array}{c}0.5 \\
(1.7)\end{array}$ & $\begin{array}{c}0.0 \\
(0.0)\end{array}$ \\
\hline Lower Back & $\begin{array}{c}4.6 \\
(18.2)\end{array}$ & $\begin{array}{c}9.3 \\
(18.1)\end{array}$ & $\begin{array}{c}7.2 \\
(26.1)\end{array}$ & $\begin{array}{c}8.8 \\
(16.3)\end{array}$ & $\begin{array}{c}0.0 \\
(0.0)\end{array}$ & $\begin{array}{c}10.0 \\
(22.4)\end{array}$ & $\begin{array}{c}3.6 \\
(10.7)\end{array}$ & $\begin{array}{c}9.5 \\
(13.9)\end{array}$ \\
\hline Hips & $\begin{array}{c}3.6 \\
(16.8)\end{array}$ & $\begin{array}{c}2.6 \\
(9.5)\end{array}$ & $\begin{array}{c}6.9 \\
(25.0)\end{array}$ & $\begin{array}{c}4.8 \\
(13.8)\end{array}$ & $\begin{array}{c}0.0 \\
(0.0)\end{array}$ & $\begin{array}{c}2.4 \\
(5.4)\end{array}$ & $\begin{array}{c}1.2 \\
(4.3)\end{array}$ & $\begin{array}{c}0.0 \\
(0.0)\end{array}$ \\
\hline Buttocks & $\begin{array}{c}3.3 \\
(17.3)\end{array}$ & $\begin{array}{c}1.6 \\
(7.1) \\
\end{array}$ & $\begin{array}{c}7.5 \\
(26.0) \\
\end{array}$ & $\begin{array}{c}3.4 \\
(10.3) \\
\end{array}$ & $\begin{array}{c}0.0 \\
(0.0)\end{array}$ & $\begin{array}{c}0.0 \\
(0.0)\end{array}$ & $\begin{array}{c}0.0 \\
(0.0)\end{array}$ & $\begin{array}{c}0.0 \\
(0.0)\end{array}$ \\
\hline Quadriceps & $\begin{array}{c}3.7 \\
(17.5)\end{array}$ & $\begin{array}{l}2.0 \\
(8.6)\end{array}$ & $\begin{array}{c}7.3 \\
(26.1)\end{array}$ & $\begin{array}{c}4.5 \\
(12.7)\end{array}$ & $\begin{array}{c}0.0 \\
(0.0)\end{array}$ & $\begin{array}{c}0.0 \\
(0.0)\end{array}$ & $\begin{array}{c}1.2 \\
(4.3)\end{array}$ & $\begin{array}{c}0.0 \\
(0.0)\end{array}$ \\
\hline Hamstrings & $\begin{array}{c}3.7 \\
(17.4)\end{array}$ & $\begin{array}{c}4.1 \\
(14.2)\end{array}$ & $\begin{array}{c}8.3 \\
(25.8)\end{array}$ & $\begin{array}{c}3.7 \\
(9.5)\end{array}$ & $\begin{array}{c}0.0 \\
(0.0)\end{array}$ & $\begin{array}{c}14.0 \\
(31.3)\end{array}$ & $\begin{array}{c}0.0 \\
(0.0)\end{array}$ & $\begin{array}{c}0.0 \\
(0.0)\end{array}$ \\
\hline Calves & $\begin{array}{c}6.7 \\
(20.2)\end{array}$ & $\begin{array}{l}11.8^{b} \\
(23.9)\end{array}$ & $\begin{array}{c}10.0 \\
(26.5)\end{array}$ & $\begin{array}{c}8.7 \\
(18.2)\end{array}$ & $\begin{array}{c}10.8 \\
(24.1)\end{array}$ & $\begin{array}{c}41.8 \\
(39.7)\end{array}$ & $\begin{array}{c}0.9 \\
(3.3)\end{array}$ & $\begin{array}{c}1.8 \\
(6.7)\end{array}$ \\
\hline Feet & $\begin{array}{c}4.5 \\
(18.1)\end{array}$ & $\begin{array}{c}6.6 \\
(15.5)\end{array}$ & $\begin{array}{c}7.0 \\
(25.2)\end{array}$ & $\begin{array}{c}4.9 \\
(10.2)\end{array}$ & $\begin{array}{c}7.8 \\
(17.4)\end{array}$ & $\begin{array}{c}21.4 \\
(30.8)\end{array}$ & $\begin{array}{c}0.0 \\
(0.0)\end{array}$ & $\begin{array}{l}1.8( \\
6.3)\end{array}$ \\
\hline Total & $\begin{array}{c}88.1 \\
(189.6) \\
\end{array}$ & $\begin{array}{c}81.8 \\
(132.0) \\
\end{array}$ & $\begin{array}{c}117.1 \\
(280.7) \\
\end{array}$ & $\begin{array}{c}86.2 \\
(130.6) \\
\end{array}$ & $\begin{array}{r}90.2 \\
(59.4) \\
\end{array}$ & $\begin{array}{c}174.4 \\
(225.7) \\
\end{array}$ & $\begin{array}{c}52.8 \\
(52.0) \\
\end{array}$ & $\begin{array}{c}34.5 \\
(23.3) \\
\end{array}$ \\
\hline
\end{tabular}






Figure 3. Mean (+s) neck pain scores for each group for loads of 0-20\% BM

\subsection{Pain scores at maximum load}

Table 3 shows pain scores at the final load in each of the conditions. Considering all participants, overall RPE scores were similar for both loading methods $(18.1 \pm 1.8,18.1 \pm 2.2$ for head and back load respectively, $\mathrm{P}=0.860$ ). Analysis of the VAS scores revealed no significant difference between the two conditions (overall pain scores $360 \pm 350$ v $433 \pm 337$ for head and back loading respectively, $\mathrm{P}=0.254)$. There was, however, a significant main effect for body area $(\mathrm{P}<0.0005)$ with the pain score associated with the neck being significantly greater than all other body areas $(\mathrm{P}<0.0005$ $-\mathrm{P}=0.003)$ except for the shoulders $(\mathrm{P}=1.000)$, upper back $(\mathrm{P}=1.000)$ and the lower back $(\mathrm{P}=0.325)$. Similarly the scores for the shoulders were significantly greater than all other body areas $(\mathrm{P}<0.005-$ $\mathrm{P}=0.034)$ except for the neck $(\mathrm{P}=1.000)$ and the upper back $(\mathrm{P}=0.263)$ whilst the scores for the upper back were significantly greater than those for all other body areas $(\mathrm{P}<0.005-\mathrm{P}=0.012)$ except for the neck, shoulders, lower back $(\mathrm{P}=1.000)$ and the quadriceps $(\mathrm{P}=0.269)$. There was also a significant interaction between loading condition and body area $(\mathrm{P}=0.003)$. The pain score for the neck was significantly higher for the head-load condition than the back-load condition (95\% CI 's $65.9-88.3$ 
and $22.8-56.7$ respectively) with the pain score for the neck whilst head-loading being the highest score recorded for any body area in any condition. Pain scores for shoulders and calves both tended to be higher in the back-load condition as opposed to head-loading (95\% CI's Shoulders: $37.7-56.6 \mathrm{v}$ $51.8-76.8$, Calves: $4.5-30.8$ v $24.2-52.3$ ). Pain scores in all other categories were higher for the back load condition than the head load condition but not significantly so (Table 3 ). There was no significant difference between the groups $(\mathrm{P}=0.122)$ and pattern of response was consistent across the group $\mathrm{x}$ body area interaction $(\mathrm{P}=0.292)$ and the loading condition $\mathrm{x}$ group interaction $(\mathrm{P}=0.959)$.

Table 3. Mean $(+s)$ pain scores at final load for each group (a indicates significant difference between conditions $(P<0.05)$, $b$ indicates trend for difference between conditions $(P<0.10))$

\begin{tabular}{|c|c|c|c|c|c|c|c|c|}
\hline & \multicolumn{2}{|c|}{ Whole Group } & \multicolumn{2}{|c|}{ EXP } & \multicolumn{2}{|c|}{ INT } & \multicolumn{2}{|c|}{ NON } \\
\hline & Head & Back & Head & Back & Head & Back & Head & Back \\
\hline RPE & $\begin{array}{l}18.1 \\
(1.8)\end{array}$ & $\begin{array}{l}18.1 \\
(2.2)\end{array}$ & $\begin{array}{l}18.0 \\
(1.8)\end{array}$ & $\begin{array}{l}18.1 \\
(2.5)\end{array}$ & $\begin{array}{l}18.0 \\
(2.1)\end{array}$ & $\begin{array}{l}17.9 \\
(2.2)\end{array}$ & $\begin{array}{l}18.4 \\
(1.8)\end{array}$ & $\begin{array}{l}18.3 \\
(2.2)\end{array}$ \\
\hline Neck & $\begin{array}{l}72.8^{\mathbf{a}} \\
(21.9)\end{array}$ & $\begin{array}{c}40.0 \\
(43.1)\end{array}$ & $\begin{array}{l}71.3 \\
(27.9)\end{array}$ & $\begin{array}{c}51.9 \\
(44.7)\end{array}$ & $\begin{array}{c}81.4 \\
(13.4)\end{array}$ & $\begin{array}{c}49.5 \\
(41.0)\end{array}$ & $\begin{array}{c}68.3 \\
(18.0)\end{array}$ & $\begin{array}{c}19.0 \\
(36.2)\end{array}$ \\
\hline Shoulders & $\begin{array}{c}53.1 \\
(36.8)\end{array}$ & $\begin{array}{l}65.3^{b} \\
(31.9)\end{array}$ & $\begin{array}{c}60.1 \\
(35.0)\end{array}$ & $\begin{array}{c}72.9 \\
(28.8)\end{array}$ & $\begin{array}{c}54.0 \\
(45.2)\end{array}$ & $\begin{array}{c}61.4 \\
(39.7)\end{array}$ & $\begin{array}{c}44.3 \\
(33.2)\end{array}$ & $\begin{array}{c}59.2 \\
(30.3)\end{array}$ \\
\hline Upper Back & $\begin{array}{c}43.5 \\
(30.2)\end{array}$ & $\begin{array}{c}46.6 \\
(22.0)\end{array}$ & $\begin{array}{c}53.9 \\
(35.3)\end{array}$ & $\begin{array}{c}50.5 \\
(11.7)\end{array}$ & $\begin{array}{c}35.8 \\
(25.5)\end{array}$ & $\begin{array}{c}48.6 \\
(19.3)\end{array}$ & $\begin{array}{c}36.9 \\
(15.3)\end{array}$ & $\begin{array}{c}40.5 \\
(27.4)\end{array}$ \\
\hline Chest & $\begin{array}{c}21.4 \\
(34.5)\end{array}$ & $\begin{array}{c}26.6 \\
(28.8)\end{array}$ & $\begin{array}{c}32.2 \\
(42.7)\end{array}$ & $\begin{array}{c}35.0 \\
(29.5)\end{array}$ & $\begin{array}{c}21.3 \\
(17.2)\end{array}$ & $\begin{array}{c}30.3 \\
(21.0)\end{array}$ & $\begin{array}{c}8.7 \\
(17.1)\end{array}$ & $\begin{array}{c}14.0 \\
(20.9)\end{array}$ \\
\hline Abdomen & $\begin{array}{c}19.1 \\
(36.4)\end{array}$ & $\begin{array}{c}29.7 \\
(37.9)\end{array}$ & $\begin{array}{c}31.0 \\
(45.5)\end{array}$ & $\begin{array}{c}39.7 \\
(43.6)\end{array}$ & $\begin{array}{l}16.50 \\
(12.1)\end{array}$ & $\begin{array}{c}25.3 \\
(25.1)\end{array}$ & $\begin{array}{c}6.9 \\
(19.6)\end{array}$ & $\begin{array}{c}21.0 \\
(30.6)\end{array}$ \\
\hline Lower Back & $\begin{array}{c}37.7 \\
(40.2)\end{array}$ & $\begin{array}{c}44.5 \\
(41.9) \\
\end{array}$ & $\begin{array}{c}37.4 \\
(45.6)\end{array}$ & $\begin{array}{c}53.3 \\
(44.9) \\
\end{array}$ & $\begin{array}{c}37.4 \\
(37.1)\end{array}$ & $\begin{array}{c}36.5 \\
(36.0)\end{array}$ & $\begin{array}{c}38.2 \\
(35.2)\end{array}$ & $\begin{array}{c}39.8 \\
(40.3)\end{array}$ \\
\hline Hips & $\begin{array}{c}19.9 \\
(36.3)\end{array}$ & $\begin{array}{c}28.4 \\
(38.7)\end{array}$ & $\begin{array}{c}30.3 \\
(44.2)\end{array}$ & $\begin{array}{c}39.2 \\
(45.2)\end{array}$ & $\begin{array}{c}16.1 \\
(11.0)\end{array}$ & $\begin{array}{c}25.8 \\
(25.9)\end{array}$ & $\begin{array}{c}10.4 \\
(24.9)\end{array}$ & $\begin{array}{c}17.5 \\
(29.5)\end{array}$ \\
\hline Buttocks & $\begin{array}{c}10.3 \\
(29.6)\end{array}$ & $\begin{array}{l}22.6 \\
(35.2)\end{array}$ & $\begin{array}{c}24.7 \\
(43.3)\end{array}$ & $\begin{array}{c}31.8 \\
(40.4)\end{array}$ & $\begin{array}{c}0.9 \\
(2.7)\end{array}$ & $\begin{array}{c}22.1 \\
(19.9)\end{array}$ & $\begin{array}{c}0.0 \\
(0.0)\end{array}$ & $\begin{array}{c}12.1 \\
(27.1)\end{array}$ \\
\hline Quadriceps & $\begin{array}{c}24.0 \\
(38.5)\end{array}$ & $\begin{array}{c}39.1 \\
(39.4)\end{array}$ & $\begin{array}{c}32.2 \\
(44.5)\end{array}$ & $\begin{array}{c}47.7 \\
(42.9)\end{array}$ & $\begin{array}{c}21.9 \\
(28.4)\end{array}$ & $\begin{array}{c}27.8 \\
(29.8)\end{array}$ & $\begin{array}{c}15.7 \\
(29.4)\end{array}$ & $\begin{array}{c}37.3 \\
(35.7)\end{array}$ \\
\hline Hamstrings & $\begin{array}{c}19.5 \\
(36.6) \\
\end{array}$ & $\begin{array}{c}32.1 \\
(39.4) \\
\end{array}$ & $\begin{array}{l}30.12 \\
(43.3)\end{array}$ & $\begin{array}{c}45.2 \\
(44.7)\end{array}$ & $\begin{array}{c}22.9 \\
(31.4)\end{array}$ & $\begin{array}{c}26.8 \\
(27.8) \\
\end{array}$ & $\begin{array}{c}4.6 \\
(15.1)\end{array}$ & $\begin{array}{c}20.6 \\
(30.6) \\
\end{array}$ \\
\hline Calves & $\begin{array}{c}20.7 \\
(36.8)\end{array}$ & $\begin{array}{l}38.9^{b} \\
(39.2)\end{array}$ & $\begin{array}{c}36.2 \\
(44.9)\end{array}$ & $\begin{array}{c}52.7 \\
(40.4)\end{array}$ & $\begin{array}{c}20.6 \\
(24.6)\end{array}$ & $\begin{array}{c}48.8 \\
(40.7)\end{array}$ & $\begin{array}{c}2.6 \\
(8.4)\end{array}$ & $\begin{array}{l}15.56 \\
(24.4)\end{array}$ \\
\hline Feet & $\begin{array}{c}18.7 \\
(34.9)\end{array}$ & $\begin{array}{c}28.94(38 \\
.1)\end{array}$ & $\begin{array}{c}32.3 \\
(42.7)\end{array}$ & $\begin{array}{c}44.0 \\
(43.9)\end{array}$ & $\begin{array}{c}19.6 \\
(15.1)\end{array}$ & $\begin{array}{c}33.1 \\
(29.4)\end{array}$ & $\begin{array}{c}2.0 \\
(6.6)\end{array}$ & $\begin{array}{c}8.1 \\
(19.0)\end{array}$ \\
\hline Total & $\begin{array}{l}360.03 \\
(350.4)\end{array}$ & $\begin{array}{c}433.3 \\
(337.3)\end{array}$ & $\begin{array}{c}471.7 \\
(458.2)\end{array}$ & $\begin{array}{c}558.5 \\
(391.2)\end{array}$ & $\begin{array}{c}345.8 \\
(165.1)\end{array}$ & $\begin{array}{c}419.9 \\
(255.3)\end{array}$ & $\begin{array}{c}238.5 \\
(120.7)\end{array}$ & $\begin{array}{c}295.0 \\
(188.4)\end{array}$ \\
\hline
\end{tabular}

\subsection{Comparison of pain scores at penultimate and final loads}

Table 4 provides a comparison of mean pain scores for the whole group at individual's penultimate and final loads. Analysis of the VAS data indicates a significant time effect with the overall pain score at the final load being significantly greater than at the penultimate load $(\mathrm{P}<0.0005)$ 
with this effect consistent across loading conditions $(\mathrm{P}=0.753)$ and body area $(\mathrm{P}=0.186)$. As

previously there were no significant main effects or interactions involving groups.

Table 4. Mean $(+s)$ differences and percentage change in pain scores for each body area between penultimate and final load

\begin{tabular}{|c|c|c|c|}
\hline & & $\begin{array}{c}\text { Mean } \\
\text { Difference }\end{array}$ & $\begin{array}{c}\text { Percentage } \\
\text { Change }\end{array}$ \\
\hline \multirow[b]{2}{*}{ RPE } & Head & $2.7(2.4)$ & 17.2 \\
\hline & Back & $2.1(2.3)$ & 12.9 \\
\hline \multirow[b]{2}{*}{ Neck } & Head & $19.6(25.4)$ & 36.8 \\
\hline & Back & $8.0(19.2)$ & 25.0 \\
\hline \multirow[b]{2}{*}{ Shoulders } & Head & $17.5(19.1)$ & 49.3 \\
\hline & Back & $12.8(20.5)$ & 24.3 \\
\hline \multirow[b]{2}{*}{ Upper Back } & Head & $13.1(17.1)$ & 43.2 \\
\hline & Back & $11.8(14.3)$ & 34.1 \\
\hline \multirow[b]{2}{*}{ Chest } & Head & $12.0(20.7)$ & 127.2 \\
\hline & Back & $6.3(10.8)$ & 31.1 \\
\hline \multirow[b]{2}{*}{ Abdomen } & Head & $6.3(15.6)$ & 49.4 \\
\hline & Back & $13.6(24.5)$ & 84.5 \\
\hline \multirow[b]{2}{*}{ Lower Back } & Head & $14.3(20.7)$ & 61.5 \\
\hline & Back & $14.5(26.7)$ & 48.5 \\
\hline \multirow[b]{2}{*}{ Hips } & Head & $7.1(19.0)$ & 55.4 \\
\hline & Back & $10.0(20.3)$ & 54.4 \\
\hline \multirow[b]{2}{*}{ Buttocks } & Head & $1.7(5.2)$ & 19.7 \\
\hline & Back & $9.6(19.4)$ & 92.3 \\
\hline \multirow[b]{2}{*}{ Quadriceps } & Head & $10.4(22.7)$ & 76.7 \\
\hline & Back & $10.8(20.8)$ & 37.9 \\
\hline \multirow[b]{2}{*}{ Hamstrings } & Head & $7.0(21.2)$ & 56.3 \\
\hline & Back & $13.7(23.2)$ & 73.9 \\
\hline \multirow[b]{2}{*}{ Calves } & Head & $3.0(8.7)$ & 16.9 \\
\hline & Back & $5.6(12.4)$ & 16.9 \\
\hline \multirow[b]{2}{*}{ Feet } & Head & $5.4(13.5)$ & 42.2 \\
\hline & Back & $6.2(17.4)$ & 27.4 \\
\hline \multirow[b]{2}{*}{ Total } & Head & $108.8(109.5)$ & 43.3 \\
\hline & Back & $116.3(129.1)$ & 36.7 \\
\hline
\end{tabular}

\subsection{Preferences and comparisons}

Comfort, Freedom of Movement and Stability was rated at the end of both the head-loading and the back-loading trials. Overall there was no difference in rating for either comfort $(4.6 \pm 1.8$ and $3.9 \pm$ 2.0 for head and back respectively, $\mathrm{P}=0.112)$ or freedom of movement $(4.7 \pm 1.7$ and $4.6 \pm 1.5$ for 
head and back respectively, $\mathrm{P}=0.837$ ). Back-loading was, however, rated higher (i.e.. better) for stability than head-loading $(4.9 \pm 1.6 \mathrm{v} 4.0 \pm 1.8, \mathrm{P}=0.018)$. There were no group interaction effects for any of the variables. Table 5 shows the results when, after the completion of the whole experiment, participants were asked to express a preference for one pack in relation to each of the parameters.

Table 5. Participant preference for each loading method

\begin{tabular}{|l|c|c|c|c|c|c|}
\hline & \multicolumn{3}{|c|}{ Comfort } & \multicolumn{2}{l|}{$\begin{array}{l}\text { Freedom of } \\
\text { Movement }\end{array}$} & \multicolumn{2}{l|}{ Stability } \\
\hline & Head & Back & Head & Back & Head & Back \\
\hline EXP & 6 & 7 & 8 & 5 & 4 & 9 \\
\hline INT & 3 & 5 & 0 & 8 & 2 & 6 \\
\hline NON & 5 & 6 & 5 & 6 & 3 & 8 \\
\hline ALL & 14 & 18 & 13 & 19 & 9 & 23 \\
\hline
\end{tabular}

Figure 4 shows the number of participants reporting discomfort in particular body areas immediately after each loading condition whilst Figure 5 shows, for each area of the body, the number of participants identifying, after the completion of the whole experiment, either head- or back-loading as being associated with the most discomfort. Back-loading was perceived, by most participants, as providing greater discomfort than head-loading for all areas of the body except the neck, where more chose head-loading, and the feet with the same number choosing each condition. 


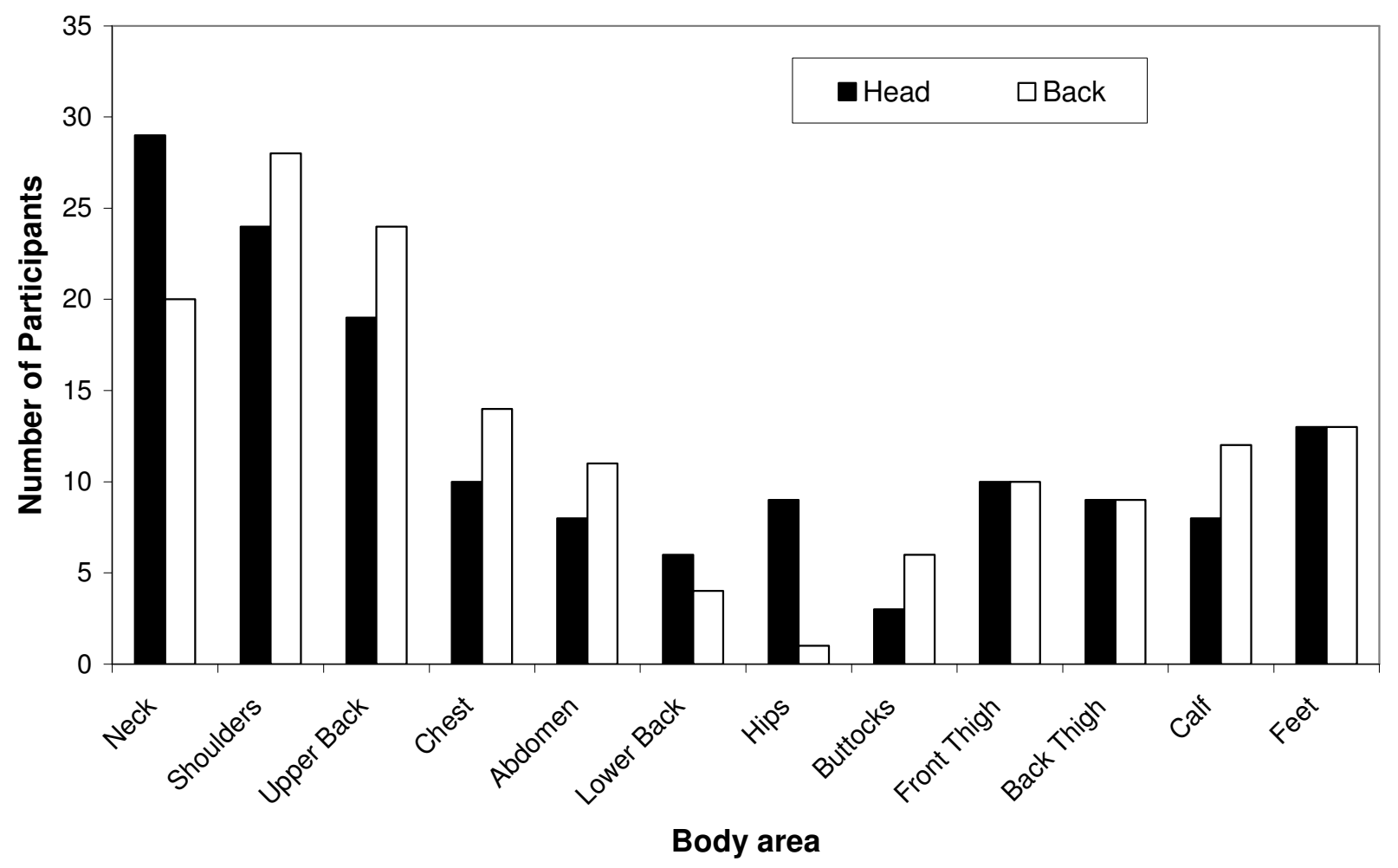

Figure 4. Post test reports of discomfort for each loading trial

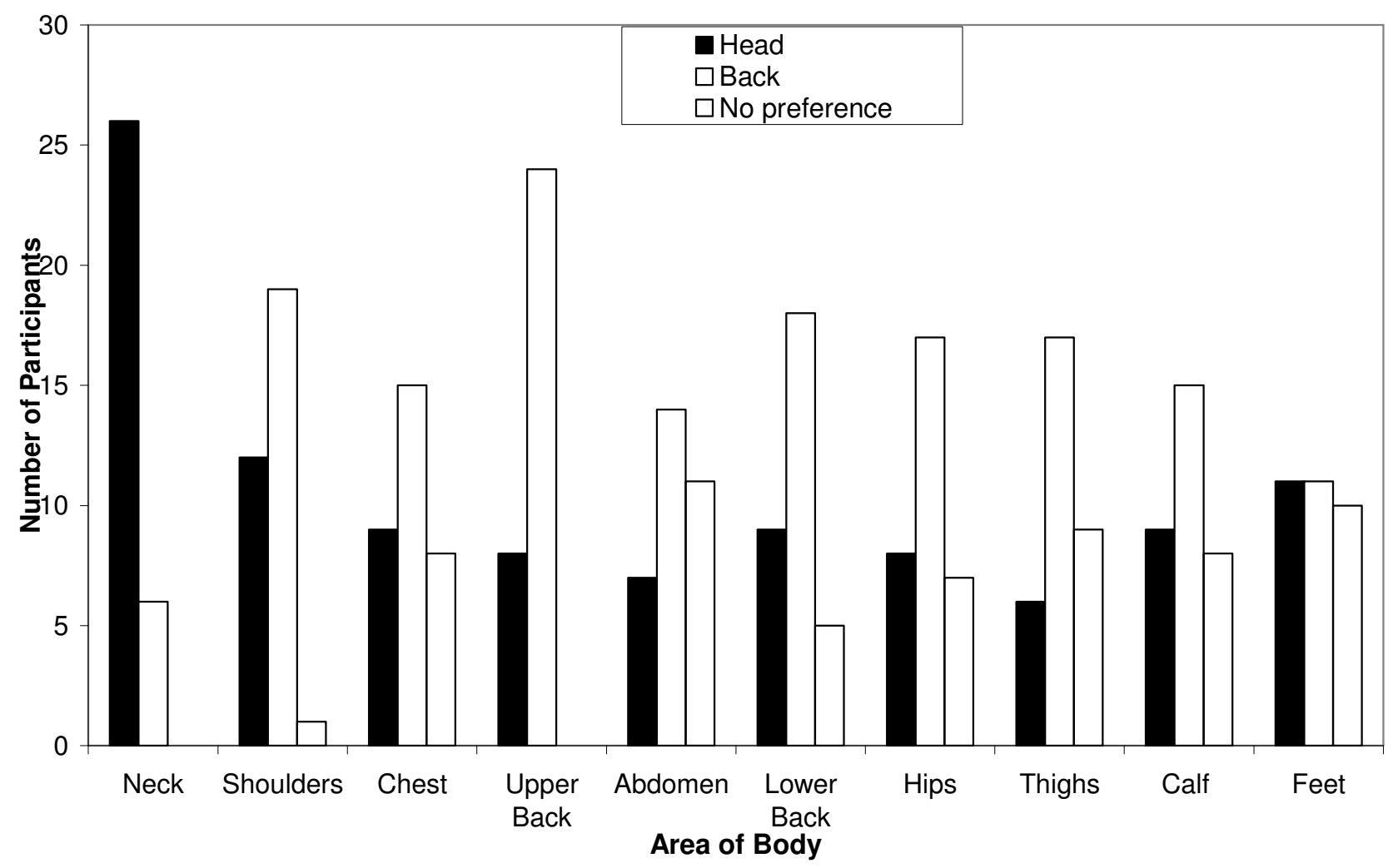

Figure 5. Number of participants indicating loading method associated with greatest discomfort for particular body areas 


\section{Discussion}

The results for the backpack condition in the present study were broadly consistent with previous findings, with the highest in-test pain scores at the final load, being associated with the neck (40 \pm 43.1), shoulders $(65.3 \pm 41.9)$, upper back $(46.6 \pm 22.0)$ lower back $(44.5 \pm 41.9)$, quadriceps $(39.1 \pm$ 39.4) and calves (38.9 \pm 39.2$)$. This is similar to Mackie et al (2003) who compared four different backpacks intended for school use $(n=12)$, employing both in-trial CRS and post trial VAS and found most reports of discomfort in the neck, shoulders and lower legs. Mackie and Legg (2008) reported a mean score of $3.3 \pm 1.4$ on a five point scale for neck and shoulder discomfort for 16 male school students carrying 15\% BM in a backpack during a simulated school day. Legg et al (1997) compared two backpacks with a $20 \mathrm{~kg}$ load $(\mathrm{n}=10)$. In-test CRS scores (10 point scale) were highest for upper back (2.1 \pm 0.9 to $3.3 \pm 0.3)$, shoulders $(3.4 \pm 1$ to $5.1 \pm 0.8)$, lower back $(1.7 \pm 0.4$ to $4.5 \pm 1.1)$ and buttocks (3.8 \pm 1 to 3.9 \pm 1.3 ). The same backpacks were also investigated in a field trial Legg et al (2003). Ten participants carried $15 \mathrm{~kg}$ (approximately 20\% BM) for fifteen minutes and reported highest discomfort scores, based on a 10 point CRS, for shoulders (2.6 \pm 1.7 to $3 \pm 2.4)$, upper back $(2.5 \pm 1.4$ to $3.6 \pm 2.4)$ and lower back $(2.9 \pm 1.5$ to $3.6 \pm 2.0)$. Perhaps the most unexpected finding was in relation to relative lack of pain and discomfort in the hip area. Backpacks with hip belts are known to be associated with relatively high levels of pain and discomfort in this area. Ling et al (2004) considered women's load carriage performance $(n=7)$ in an extended load carriage task of 56 minutes duration, at a variety of loads. Their results indicated consistent reports of discomfort over the anterior superior iliac spines and iliac crests area for the two highest loads (18.2 and $22.7 \mathrm{~kg})$. Similarly Knapik et al (1997) reported significant hip discomfort scores for 15 male soldiers after 20km marches carrying 34,48 or $61 \mathrm{~kg}$ whilst Birell and Haslam (2009) reported significantly greater hip discomfort scores for women as opposed to men in a large sample of 127 ( 29 female) after a 6.4 $\mathrm{km}$ march carrying between 20 and $25 \mathrm{~kg}$. In all these previous studies, however, the loads were relatively large and the duration was much longer than the 4 minute interval reported here The current data would suggest that the discomfort in the hips is only really associated with relatively heavy loads - the hip area saw the one of the greatest relative increases between penultimate and final load and the 
hip was also one of the body areas, along with the chest, buttocks and feet that did not see a consistent rise in VAS scores until the $15 \%$ load and beyond..

Considering the post test observations of the participants, a majority of the participants reported discomfort in the neck $(62.5 \%)$, shoulders $(87.5 \%)$ and upper back $(75 \%)$ This is again supported by the available literature. Whitfield et al (2005) surveyed 140 New Zealand school students who carried backpacks. Reports of pain or discomfort where as follows: Neck 44.3\%, Shoulders 57.9\%, Upper arms 15.7\%, Lower arms 7.1\%, Upper back 36.4\%, Lower back 35\%, hips/thighs 6.4\%, Knees 5.7\%, lower legs 5.7\%. Birrell and Hooper (2007) reported data relating to sites of discomfort after a two hour speed march carrying $20 \mathrm{~kg}$ indicating that nine out of the ten participants reported pain in the shoulders whilst five reported neck pain. Similarly, Mackie and Legg (2008) reported that $50 \%$ of the participants indicated neck and shoulder pain.

Turning to the data for the head-load condition, there is no directly comparable data available in the literature. Most closely related is Balogun et al (1986) who studied ten participants carrying loads either on the head or using a transverse or frontal yoke and reported differentiated RPE scores, relating to the neck/scalp, of $15.3 \pm 1.06$ which is consistent with the present study. It is worth noting that in the present study, as with many previous studies including Balogun et al (1986), whole body RPE was not sensitive enough to differentiate between loading conditions.

When comparing the two load carriage methods based on the data presented here, three areas of the body seem particularly important: the neck, the shoulders and the calves with the former being the key area of discomfort in head-loading and the latter two being most significant in relation to backloading. The in-test score of $72.8 \pm 21.9$ for the neck at the final load in the head-loading condition was the highest pain score for any body area in either condition and was significantly higher than that associated with back-loading $(40.0 \pm 43.1)$. Similarly, twenty six of the thirty two participants identified head-loading as being associated with more neck pain than back-loading, whilst twenty nine participants identified the neck as a source of discomfort immediately after the head-loading trial. It seems that this discomfort in the neck transcended all other variables and was, in all cases, the cause 
of early termination of the head-loading trials. Most participants indicated the back-load condition was associated with greater pain/discomfort than head-loading for all other body areas yet most participants indicated a preference for the back-load condition in terms of comfort. Further evidence of the prevalence of neck pain as a major source of discomfort was provided by the interviews with the head-loaders. They consistently reported neck pain as a result of carrying water and reported regular occasions when mothers/grandmothers, with even more experience of head-loading than the participants here, had requested neck massages when returning from water carrying. This may be indicative of the potential for chronic problems associated with head-loading that may be worthy of further research and may well be consistent with the First International African Conference on Gender, Transport and Development 2006, which declared: "Head-load carrying is detrimental to health" (http://www.buanews.gov.za/view.php?ID=06082808451002\&coll=buanew06). The high scores for the shoulder during back-loading would have been expected based on the previous studies outlined above. The pain associated with the calves has, however, been less consistently reported. Abe et al (2004) argued that the mechanics of back-loading involve an interplay between rotative torque of the centre of mass and 'excessive burden on the lower extremities' and it may well be this 'excessive burden' which is being reflected here. Examination of the data for the final two loads shows an increasing contribution of the lower limbs to the overall pain score in both conditions although the effect is more marked for the back-loading condition. Clearly some of this increase will simply be a result of fatigue as a consequence of increasing walking time. It is, however, known that loading the back provokes increased forward lean (Lloyd and Cooke, 2000) so it is plausible to assume that the disproportionate increase in lower body pain scores associated with back-loading may well be a consequence of accommodations to the increasing forward lean with increasing loads. Charteris et al (1989b) suggested that head-loading might reduce the braking phase of foot contact and increase the propulsive phase, thus it is also possible that the difference observed here between head-loading and back-loading for calf discomfort be a consequence of this, presumably by a decrease in eccentric activity. Most likely it is a consequence of both a small increase in activity associated with backloading and a small decrease in activity associated with head-loading. It should also be noted, 
however, that the differences in pain scores for the calf are perhaps not as generalisable as some of the other findings - analysis of the two questionnaires indicated that there were only nine reports of pain or discomfort in the calves after each of the experimental conditions and less than half the group, 15 indicated that back-loading provoked more discomfort in the calves than head-loading.

The general pattern of response is evident from both the analysis of loads of $0-20 \% \mathrm{BM}$ and the comparison of the scores associated with the penultimate and final loads, with increasing scores for nearly all variables with increasing load. Again this is consistent with the literature. It must be remembered, however, that, in the case of the final and penultimate data, these scores are associated with differing relative loads, ranging from $15-60 \% \mathrm{BM}$ for the penultimate trial and $20-70 \% \mathrm{BM}$ for the final trial and that 16 of the participants carried more load on the back than on the head, seven carried the same in both conditions and nine carried more on the head than on the back. Consequently, in general, the penultimate and final loads reflect greater loadings on the back than on the head. Data for the same relative load, $20 \% \mathrm{BM}$, is also presented and there remains a significant difference for neck scores and a trend for difference in calf pain scores between the two loading conditions. All of the data presented exhibits high degrees of variability as indicated by the standard deviations. This is also consistent with previous findings and is a consequence of a number of factors including individual differences in pain thresholds as well as individual differences in interpretation of the scales and likelihood of using extreme values. The data associated with the $20 \%$ load exhibits greater variability than either the penultimate or final load data. This is a consequence of the different relative intensity for individual participants, for some it was at or close to their maximum, in one or both conditions, whilst for others it was relatively light.

One particularly striking, and unexpected, finding, was the consistency of response, in all variables, across levels of experience. In all of the data analysed there was only one significant body area $\mathrm{x}$ group interaction. This occurred at loads in the 0-20\% BM range and involved the INT group. This is readily explained by the fact that for three of the eight participants in the INT group, $20 \%$ BM was a final load and consequently pain scores were relatively higher. The lack of interaction between group and method at any level for neck pain was particularly unexpected. It has previously been 
argued (Maloiy et al, 1986) that exposure to head-loading whilst young may lead to structural changes in the spine that allow very heavy loads to be carried on the head, reporting that amongst their group of five head-loaders loads of $70 \% \mathrm{BM}$ were carried with ease. During interviews at the end of the present study with participants in the EXP group, these experienced head-loaders all reported a similar process by which they had learned to carry loads on their heads. The process was essentially trial and error based on observation of older female relatives, beginning with light loads of 2-5 litres of water at 6-8 years of age. By that age of 10-12 years all the women were carrying a 20 litre bucket of water and would do so as often as three times per day. Thus it might have been expected that the experienced head-loaders would not only suffer less neck pain than their inexperienced counterparts but would also be able to carry much heavier loads. This was not the case. There were no significant differences between the groups for either neck pain or load carried. Indeed the NON group not only had the lowest neck pain score at final load, $68.3 \pm 18.0$ v $71.3 \pm 27.9$ for the EXP group, but also five of the eleven in this group carried more on their heads than on their backs as opposed to two in the EXP group although the mean maximum load for the EXP group was $29.2 \mathrm{~kg}$ as opposed to 25.5 for the NON group, a non-significant difference $(\mathrm{P}=0.595)$. It seems therefore that long term habituation to head-loading neither reduces the pain associated with it nor increases the amount of load that can be carried, although it is possible that in a less controlled environment, walking on uneven terrain, the habituated head-loaders may have an advantage in terms of balance and stability.

When asked to compare the loading conditions there was general preference for back-loading as opposed to head-loading with the main difference being related to feelings of stability. Twenty three of the women rated back-loading as more stable than head-loading, a consistent finding across all three groups. Back-loading was also generally preferred in relation to both freedom of movement and comfort, although slightly more of the EXP group indicated that head-loading allowed greater freedom of movement, presumably as a consequence of the (unusual) constriction of the shoulders associated with the backpack. Most of the women in the study had experience of carrying on their backs but not usually in a backpack as the most common load was a child. Children up to five years of age are regularly carried on the back by way of the traditional method of attachment which is via a 
blanket tied around the abdomen. The only load regularly carried in a back-pack was schoolbooks and thus the heavier loads used in this study may well have invoked greater pressure on the shoulders than had previously been experienced.

It was clear from the interviews with the EXP group that head-loading was a necessity rather than a choice. The most common loads carried are either water or firewood and neither lends themselves easily to being transported by existing load carriage systems, especially when they must usually be transported over very uneven terrain. Some of the women had recently moved to the city and reported that they had completely ceased head-loading other than when they returned to the rural areas on vacation, furthermore they indicated that, given a choice, they would not carry loads on their head, opting for a backpack instead. This choice was predicated on the beliefs that it was not only less painful but also more socially acceptable in an urban environment. 


\section{Conclusion}

The present study indicates that back-loading has certain important advantages over headloading. Most particularly it is possible to carry greater loads on the back than on the head and it is generally deemed to be more comfortable. Head-loading is characterised by significant neck pain and long term habituation does not seem to provide protection against this. Moreover the level of neck pain invoked is great enough to outweigh the fact that back-loading seems to be associated with greater discomfort in most other areas of the body. It is suggested that there is a need for further research into viable alternatives to head-loading for rural dwelling African women.

\section{Acknowledgements}

This work was funded by:

The Carnegie Trust for Higher Education in Scotland

The School of Social and Health Sciences, University of Abertay Dundee

The Department of Sports Management, Cape Peninsula University of Technology 


\section{References}

Abe, D., Muraki, S. and Yasukouchi, A. (2008) Ergonomic effects of load carriage on energy cost of gradient walking. Appl Ergon 35: 329-335

Balogun, J.A., Robertson, R.J., Goss, F.L., Edwards, M.A., Cox, R.C. and Metz, K.F.. (1986)

Metabolic and perceptual responses while carrying external loads on the head and by yoke.

Ergonomics 29(12): 1623-1635

Bastien, G.J., Willems, P.A., Schepens, B. and Heglund, N.C. (2005) Effect of load and speed on the energetic cost of walking. Eur J App Phys 94: 76-83

Birrell, S.A. and Haslam, R.A., (2008). Subjective Skeletal Discomfort Measured Using a Comfort Questionnaire Following a Load Carriage Exercise, Military Medicine, 174: 177-182

Birrell, S.A. and Hooper, R.H. (2007) Initial subjective load carriage injury data collected with interviews and questionnaires. Mili Med. 172(3): 306-311

Charteris, J., Nottrodt, J.W. and Scott, P.A. (1989a) The 'free ride' hypothesis: a second look at the efficiency of African women headload carriers. S Afr J Sci 85:68-71

Charteris, J., Scott, P.A. and Nottrodt, J.W. (1989b) Metabolic and kinematic responses of African women headload carriers under controlled conditions of load and speed. Ergonomics 32:1539-1550 Das, S.K. and Saha, H. (1966) Climbing efficiency with different modes of load carriage. Ind J Med Res 54: 866-871

Datta, S.R. and Ramanathan, N.L. (1971) "Ergonomic comparison of seven modes of carrying loads on the horizontal plane." Ergonomics 14(2):269-278

Hinnant, D.W. (1994) Psychological Evaluation and Testing in: Tollison, C.D. (ed) Handbook of Pain Management ( $2^{\text {nd }}$ Ediition) Williams and Wilkins.

Holewijn, M. (1990) Physiological strain due to load carrying. Eur J App Phys 61: 237-245

Kirk, J and Schneider, D.A. (1992) Physiological and perceptual responses to load carrying in female subjects using internal and external frame backpacks. Ergonomics 35: 445-455

Knapik, J.J, Ang, P., Meiselman, H., Johnson, W., Kirk, J., Bensel, C. and Hanlon, W. (1997). Soldier performance and strenuous road marching: Influence of load mass and load distribution. Military

Medicine, 162: 62-67

LaFiandra, M, Wagenaar, RC, Holt, KG and Obusek, JP (2003). How do load carriage and walking speed influence trunk coordination and stride parameters? J Biomech 36: 87-95

Legg, S.J, Perko, L. and Campbell, P. (1997) Subjective perceptual methods for comparing backpacks. Ergonomics 40(8): 809-817.

Legg, S.J, Barr, A.A., and Hedderley, D.I. (2003) Subjective perceptual methods for comparing backpacks in the field. Ergonomics 46(9): 935-955.

Ling, W., Houston, V., Yung-Shen, T., Chui, K. and Kirk, J. (2004) Women's load carriage performance using modular lightweight load carrying equipment. Mil Med 169(11): 914-919 Lloyd, R. and Cooke, C.B. (2000) Relationships between physiological and postural adjustments to load carriage for rucksack designs. J Sports Sci 18: 25-26

Mackie, H.W. and Legg, S.J, (2008) Postural and subjective responses to realistic schoolbag carriage. Ergonomics 51(2): 217-231

Mackie, H.W., Legg, S.J, Beadle, J., and Hedderley, D. (2003) Comparison of four different backpacks intended for school use. App Ergon 34: 257-264

Maloiy, G.M., Heglund, N.C., Prager, L.M., Cavagna, G.A. and Taylor, C.R. (1986) Energetic costs of carrying loads: have African women discovered an economic way? Nature 319 668-669

Malvill, N.J., Burns, W.C., LimHa and Basnyat, R. (2001) Commercial porters of Eastern Nepal: health status, physical work capacity and energy expenditure. Am J Hum Biol 13: 44-56

Minetti, A.E., Formenti, F. and Ardigo, L.P. (2006) Himalayan porter's specialization: metabolic power, economy, efficiency and skill. Proc Biol Soc 273: 2791-2797

Nag, P.K. and Sen, R.N. (1978) Cardiorespiratory performance of porters carrying loads on a treadmill. Ergonomics 22: 897-907

Sharpe, SR, Holt, KG, Saltzman, E, and Wagenaar, RC (2008). Effects of hip belt on transverse plane trunk coordination and stability during load carriage. J Biomech 41: 968-976 
Stuempfle, K.J., Drury, D.G.and Wilson, A.L. (2004) Effect of load position on physiological and perceptual responses during load carriage with an internal frame backpack. Ergonomics 47(7): 784789

Whitfield, J.., Legg, S.J, and Hedderley, D.I (2005) Comparison of four different backpacks intended for school use. Appl Ergon 36: 193-198 